\title{
Dose recalculation in megavoltage cone-beam CT for treatment evaluation: Removal of cupping and truncation artefacts in scans of the thorax and abdomen
}

Citation for published version (APA):

Petit, S. F., van Elmpt, W. J. C., Lambin, P., \& Dekker, A. L. A. J. (2010). Dose recalculation in megavoltage cone-beam CT for treatment evaluation: Removal of cupping and truncation artefacts in scans of the thorax and abdomen. Radiotherapy and Oncology, 94(3), 359-366.

https://doi.org/10.1016/j.radonc.2009.12.001

Document status and date:

Published: 01/03/2010

DOI:

10.1016/j.radonc.2009.12.001

Document Version:

Publisher's PDF, also known as Version of record

Document license:

Taverne

Please check the document version of this publication:

- A submitted manuscript is the version of the article upon submission and before peer-review. There can be important differences between the submitted version and the official published version of record. People interested in the research are advised to contact the author for the final version of the publication, or visit the DOI to the publisher's website.

- The final author version and the galley proof are versions of the publication after peer review.

- The final published version features the final layout of the paper including the volume, issue and page numbers.

Link to publication

\footnotetext{
General rights rights.

- You may freely distribute the URL identifying the publication in the public portal. please follow below link for the End User Agreement:

www.umlib.nl/taverne-license

Take down policy

If you believe that this document breaches copyright please contact us at:

repository@maastrichtuniversity.nl

providing details and we will investigate your claim.
}

Copyright and moral rights for the publications made accessible in the public portal are retained by the authors and/or other copyright owners and it is a condition of accessing publications that users recognise and abide by the legal requirements associated with these

- Users may download and print one copy of any publication from the public portal for the purpose of private study or research.

- You may not further distribute the material or use it for any profit-making activity or commercial gain

If the publication is distributed under the terms of Article 25fa of the Dutch Copyright Act, indicated by the "Taverne" license above,

Download date: 26 Apr. 2023 
Cone-beam CT

\title{
Dose recalculation in megavoltage cone-beam CT for treatment evaluation: Removal of cupping and truncation artefacts in scans of the thorax and abdomen
}

\author{
Steven F. Petit*, Wouter J.C. van Elmpt, Philippe Lambin, André L.A.J. Dekker \\ Department of Radiation Oncology (MAASTRO Clinic), Maastricht University Medical Center (MUMC+), Maastricht, The Netherlands
}

\section{A R T I C L E I N F O}

\section{Article history:}

Received 29 July 2009

Received in revised form 2 December 2009

Accepted 6 December 2009

Available online 7 January 2010

\section{Keywords:}

Megavoltage cone-beam CT (MVCBCT)

Cupping artefacts

Truncation artifacts

Dose recalculation

Dose reconstruction

Treatment evaluation

\begin{abstract}
A B S T R A C T
Purpose: To correct megavoltage cone-beam CT (MVCBCT) images of the thorax and abdomen for cupping and truncation artefacts to reconstruct the 3D-delivered dose distribution for treatment evaluation. Materials and methods: MVCBCT scans of three phantoms, three lung and two rectal cancer patients were acquired. The cone-beam projection images were iteratively corrected for cupping and truncation artefacts and the resulting primary transmission was used for cone-beam reconstruction. The reconstructed scans were merged into the planning CT scan (MVCBCT+). Dose distributions of clinical IMRT, stereotactic and conformal treatment plans were recalculated on the uncorrected and corrected MVCBCT+ scans using the treatment planning system and compared to the planned dose distribution.

Results: The dose distributions on the corrected MVCBCT+ of the phantoms were accurate for 99\% of the voxels within $2 \%$ or $2 \mathrm{~mm}$. Using this method the errors in mean GTV dose reduced from about $10 \%$ to $1 \%$ for the patients.

Conclusions: The method corrects cupping and truncation artefacts in cone-beam scans of the thorax and abdomen in addition to head-and-neck (demonstrated previously). The corrected scans can be used to calculate the influence of anatomical changes on the 3D-delivered dose distribution.
\end{abstract}

(c) 2009 Elsevier Ireland Ltd. All rights reserved. Radiotherapy and Oncology 94 (2010) 359-366
With the introduction of kilovoltage and megavoltage conebeam CT ( $\mathrm{kVCBCT}$ and MVCBCT), image guided radiotherapy (IGRT) strategies have become available in a growing number of institutes [1-3]. An attractive next step is to use these images to adapt the treatment if necessary [4]. However, the importance of anatomical changes or set-up errors, observed during treatment, in terms of dose to the target and healthy organs is often difficult to interpret and it can be complex to decide when and how to replan [5]. A vision that is gaining in popularity is to reconstruct and accumulate the $3 \mathrm{D}$ dose distribution delivered to the patient during the previous treatment fractions [6,7]. The delivered dose to the target and normal tissues can then be reviewed and could give the physician more insight in whether and how the treatment plan should be adapted. Moreover, analysis of the delivered dose distribution in a large cohort of patients could help to establish objective rules to guide treatment interventions based on observed anatomical changes without reconstructing the 3D dose distribution for every patient [8].

It has been shown that the energy fluence of the treatment beams exiting the patient can be measured with an electronic

\footnotetext{
* Corresponding author. Address: Department of Radiation Oncology (MAASTRO Clinic), Maastricht University Medical Center (MUMC+), GROW - School for Oncology and Developmental Biology, Dr. Tanslaan 12, 6201 BN, Maastricht, The Netherlands.

E-mail address: steven.petit@maastro.nl (S.F. Petit).
}

portal imaging device (EPID) and back-projected through a MVCBCT scan of the patient, acquired moments before treatment. The 3D dose distribution delivered to the patient can then be reconstructed [9-12]. MVCBCT scans suffer from cupping artefacts and for objects larger than the field-of-view (FOV) of the MVCBCT scanner, also from truncation artefacts. Hence, uncorrected MVCBCT scans are in general not suitable for accurate dose calculations. Various groups have demonstrated that these artefacts can be removed [13-19]. Strategies were proposed to correct the MVCBCT portal images for scatter radiation and beam hardening before cone-beam reconstruction $[13,14,16,17]$. Others removed the cupping artefacts of MVCBCT scans of the head-and-neck or abdomen after cone-beam reconstruction, using predefined ellipsoid-shaped correction factors $[15,18]$. The planning CT scan can also be registered to the MVCBCT scan to derive accurate electron density (ED) values [19,20]. Advantages of cupping correction methods that correct the portal images are that they can be fully automated without making use of any prior knowledge about the geometry of the scanned object and do not require deformable registration. A disadvantage is that these methods cannot directly solve truncation artefacts caused by attenuation of the beam outside the FOV.

Most of the research has focused on MVCBCT images of the abdomen or head-and-neck. Although MVCBCT has been used before to image lung cancer patients [21,22], to the best of our knowledge, none of the cupping correction methods have been 
described for MVCBCT scans of the thorax. This is the most complex treatment site for cupping correction because of the large inhomogeneities and interfaces at the lung, bone and soft tissue boundaries. Moreover, truncation artefacts occur because the thorax cannot be encompassed by the field-of-view of the MVCBCT scanner without shifting the detector, which is not yet possible for most of the current clinical MVCBCT scanners.

The goal of the current study was to develop a method to correct cupping and truncation artefacts of MVCBCT scans of the thorax and abdomen and calibrate the scans to ED such that they are suitable for accurate dose calculations. A previously proposed cupping correction method for head-and-neck was extended to deal with truncation artefacts that occur when objects are larger than the FOV of the MVCBCT image. The method does not require patient or phantom-specific parameters or deformable registration. Validation was performed with MVCBCT scans of homogeneous and anthropomorphic phantoms. Dose recalculation using the corrected MVCBCT scans was demonstrated for three lung and two rectal cancer patients, including two patients with large deformations between the planning CT and MVCBCT.

\section{Materials and methods}

\section{Hardware and operation}

Two Siemens Oncor medical linear accelerators (linacs) with OptiVue 1000 ST amorphous silicon EPIDs $(1024 \times 1024$ pixels, $41 \times 41 \mathrm{~cm}^{2}$ corresponding to a field size in the isocenter plane of $27.4 \times 27.4 \mathrm{~cm}^{2}$ ) and MVCBCT acquisition mode were used (Siemens Medical Solutions, Concord, CA, USA). The MVCBCT acquisition procedure was described in detail previously [16,23]. Before MVCBCT reconstruction, the portal images were filtered with a Perona-Malik diffusion filter, implemented in MevisLab (MevisLab, University of Bremen, Germany), corrected for differences in accelerator output and divided by the portal images measured without an object in the beam, to yield the transmission images. The transmission images were reconstructed to a 3D scan using a research software package (Siemens Medical Solutions, Concord, CA, USA). kV CT scans were made with Siemens CT Open scanners (Siemens Medical Solutions, Erlangen, Germany) and the Hounsfield Units were converted to electron density (ED) using our clinically applied conversion tables.

\section{Origin of the artefacts}

The filtered back-projection algorithm used for cone-beam reconstruction assumes that the log of the measured transmission, $T$, is linear with the radiological path length given by

$\ln (T)=-\mu_{w} t_{\text {rad }}$

with $\mu_{w}$ being the attenuation coefficient in water and $t_{\text {rad }}$ being the radiological path length projected from the source to a point on the detector. For a mono-energetic MV photon pencil beam this relation holds. However, for MVCBCT the measured transmission is the sum of the primary and scatter transmission, the beam is polyenergetic with an energy spectrum that varies with off-axis position and the EPID response is energy-dependent. These phenomena cause the cupping artefacts. Truncation artefacts occur when the object is larger than the FOV of the MVCBCT. Attenuation of the beam in the part of the object outside the FOV is reconstructed as if it had occurred within the FOV, leading to overestimations in ED near the boundaries of the FOV.

\section{Correction algorithm}

The proposed correction procedure is shown in Fig. 1. The radiological path length of the beam through the object at a given gantry angle can be extracted from the measured transmission as described previously [16]. The attenuation of mono-energetic raylines depends only on their radiological path length. Therefore the attenuation that occurs outside the FOV of the MVCBCT is directly related to the radiological path length of the beam outside the FOV. Thus by subtracting the latter from the total radiological path length, the attenuation of the beam that occurs within the part of the object that is inside the FOV of the MVCBCT can be calculated. The corresponding primary transmission of mono-energetic raylines is calculated using (1). This procedure is repeated for the 200 transmission images and the derived primary transmission images are used for MVCBCT reconstruction.

The radiological path length of the beam outside the FOV cannot be derived using the MVCBCT scan. Instead it was calculated using the planning CT scan. First, the planning CT scan was registered to the MVCBCT scan. Then the ED of the part of the CT that corresponds to the FOV of the MVCBCT was set to 0 (Fig. 1). By raytracing through the adapted planning $\mathrm{CT}$, the radiological path length distribution of the beam outside the FOV of the MVCBCT was derived for each gantry angle.

\section{Calibration to electron density and stitching the missing tissue}

The CT numbers of the reconstructed MVCBCT scans were calibrated to ED using a linear relationship. The slope and intercept were fitted by applying the method to a MVCBCT of a cylinder with a radius of $10 \mathrm{~cm}$ filled with water and by setting the mean value within the cylinder to an ED of 1 and outside the cylinder to 0 .

For dose calculations, the patient's entire anatomy is needed, also outside the FOV of the MVCBCT. Therefore the missing part of the patient's anatomy was taken from the planning CT and stitched to the MVCBCT scan using the derived registration matrix. The combined scan was referred to as the MVCBCT+ in analogy with Aubry et al. [19].

\section{Verification and dose calculation}

The method was tested by analyzing dose distributions of clinical treatment plans that were recalculated on the uncorrected and corrected MVCBCT+ and on the planning CT scan for comparison. Our clinical treatment planning system (TPS) XiO 4.3.4 (CMS, St. Louis, MO) was used with the superposition algorithm and a grid size of $3 \times 3 \times 3 \mathrm{~mm}^{3}$. The dose distributions were compared using $\gamma$ analysis $(2 \%, 2 \mathrm{~mm})$ within the $20 \%$ iso-dose contours on transversal slices through the isocenter and by calculating the mean dose in different volumes of interest (VOIs). ED values are presented relative to the ED of water. The window level settings of the presented CT and MVCBCT images were 1.0 \pm 0.5 ED (Center \pm Width) unless mentioned otherwise.

\section{Phantom evaluation}

For the phantom study, MVCBCT scans were acquired of three phantoms with a total dose of $52 \mathrm{MU}$.

The first phantom was a water equivalent CIRS thorax phantom with lung ( $E D=0.207)$ and bone $(E D=1.506)$ inserts positioned along the rotational axis of the linac (Model 002LFC, Computerized Imaging Reference Systems Inc. Norfolk, VA, USA). Dose calculations were performed for a single $6 \mathrm{MV} 5 \times 5 \mathrm{~cm}^{2}$ photon beam through the isocenter in the left-right direction. In addition, a clinical IMRT treatment plan with seven $10 \mathrm{MV}$ photon beams was recalculated on the MVCBCT+. The dose is reported in the lung and bone equivalent regions and in four VOIs inside the water equivalent material. The first VOI was a cylindrical volume defined in the center along the radial axis with a diameter and length of 

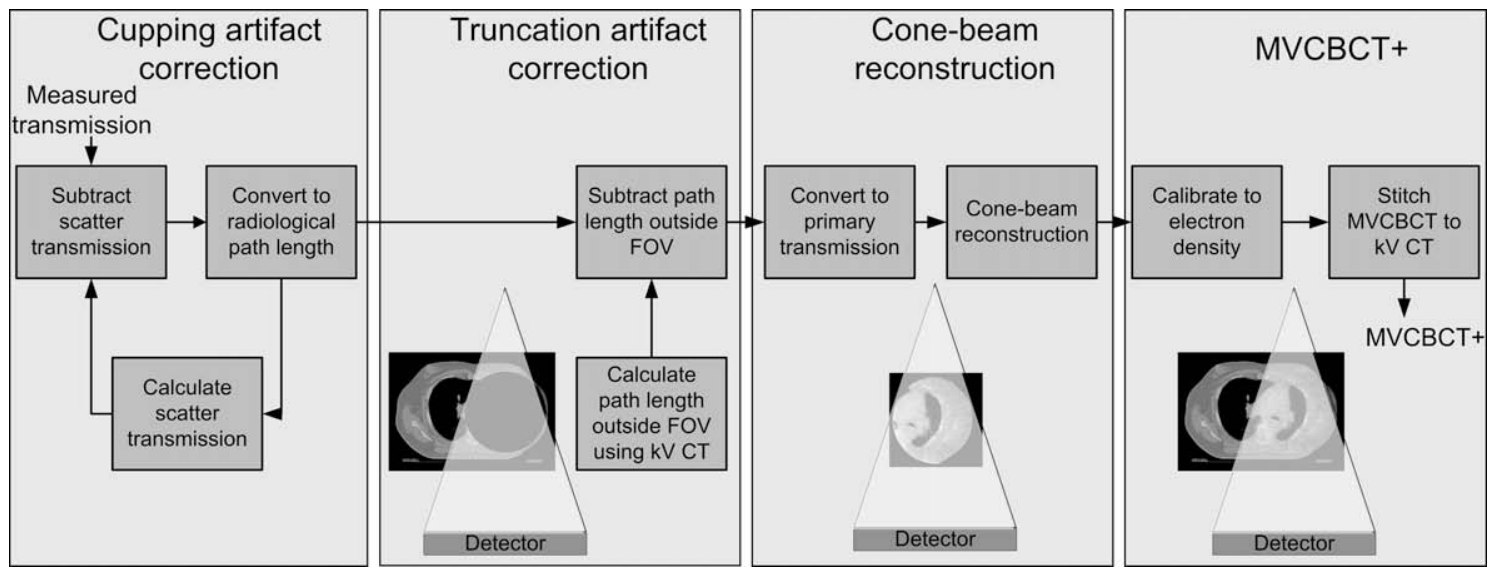

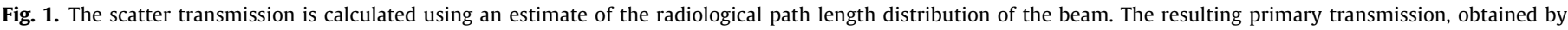

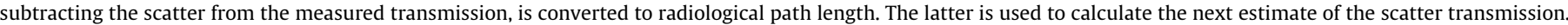

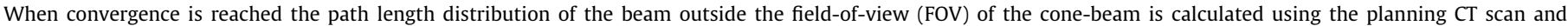

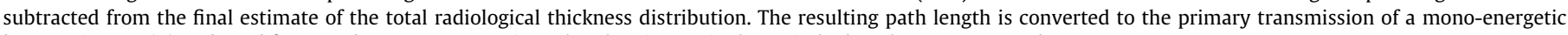
beam using Eq. (1) and used for cone-beam reconstruction. The planning CT is then stitched to the reconstructed MVCBCT scan.

$4 \mathrm{~cm}$. The subsequent VOIs were generated by isotropically expanding the previous VOI with $1 \mathrm{~cm}$.

Secondly, the Rando thorax phantom (Radiology Support Devices Inc., Ramsey, NJ, USA) was used in combination with a stereotactic body radiotherapy (SBRT) plan with four $6 \mathrm{MV}$ and five 10 MV beams.

The third phantom was a CIRS IMRT homogeneous phantom (Model 002H5) in combination with a clinical IMRT prostate plan with five $10 \mathrm{MV}$ photon beams. Eight cylindrical VOIs were defined as described above with diameters varying between 4 and $11 \mathrm{~cm}$.

\section{Patient evaluation}

\section{Lung cancer patients}

Two lung cancer patients were treated with SBRT with, respectively, eight (10 MV) and eleven ( 6 and $10 \mathrm{MV}$ ) photon beams in three fractions of $18 \mathrm{~Gy}$ ( $54 \mathrm{~Gy}$ total). 4DCT scans were acquired and the $50 \%$ maximum exhale phases were used for treatment planning. MVCBCT scans of $13 \mathrm{MU}$ were acquired at the first treatment fraction. Lung cancer patient 1 had a T1NOM0 squamous cell carcinoma in the left upper lobe with a PTV of $58 \mathrm{~cm}^{3}$. Lung cancer patient 2 had a T2NOMO adeno carcinoma in the left lower lobe about $2 \mathrm{~cm}$ from the diaphragm with a PTV of $155 \mathrm{~cm}^{3}$.

Lung cancer patient 3 had a T1N3M0 carcinoma with involved mediastinal lymph nodes and a PTV of $549 \mathrm{~cm}^{3}$. This patient was treated with a 3D conformal plan with three $10 \mathrm{MV}$ beams and a total dose of $59 \mathrm{~Gy}(30 \times 1.5 \mathrm{~Gy}$ followed by $7 \times 2 \mathrm{~Gy})$. A MVCBCT scan of 7 MU was acquired at fraction 17.

\section{Rectal cancer patients}

Two rectal cancer patients were treated with 3D conformal plans with 4 beams with a total dose of $25 \mathrm{~Gy}$ given in 5 fractions. MVCBCT scans of $13 \mathrm{MU}$ were acquired during therapy. Rectal cancer patient 1 had a T3NOMO carcinoma and a PTV of $969 \mathrm{~cm}^{3}$. Rectal cancer patient 2 had a T3NOMx carcinoma and a PTV of $1006 \mathrm{~cm}^{3}$. All phantoms and patients were larger than the FOV of the MVCBCT.

\section{Sensitivity analysis}

During the course of therapy, anatomy changes might occur outside the FOV of the MVCBCT, e.g. because of breathing motion or weight loss. These changes could affect the removal of truncation artefacts and lead to errors in dose. We simulated the effect of breathing motion by stitching the corrected MVCBCT of lung cancer patient 2 into the maximum inhale and the maximum exhale phase of the 4D CT scan using the same registration matrix. The dose distributions of the treatment plan were recalculated in both MVCBCT+ and compared.

The effect of weight loss was simulated using a virtually generated MVCBCT of a water cylinder with a diameter of $30 \mathrm{~cm}$ that was corrected for truncation artefacts based on a virtual $\mathrm{kV} \mathrm{CT}$ of a cylinder with a diameter of $33.3 \mathrm{~cm}$. This situation corresponded to a MVCBCT, acquired during treatment after the patient lost weight, which was corrected for truncation artefacts using the original planning CT. The amount of weight lost was equivalent to a decrease in patient diameter of $10 \%$. The corrected MVCBCT images were stitched into the virtual kV CT scan to generate the MVCBCT+. Dose calculations were performed with a box technique of four $5 \times 5 \mathrm{~cm}^{2} 6 \mathrm{MV}$ beams.

\section{Results}

The dose distributions of the originally planned beams were recalculated on the uncorrected and corrected MVCBCT+ using the clinical TPS and compared to the planned dose distributions at the planning CT scan.

\section{Phantoms}

Fig. 2 shows the reconstructed ED in the uncorrected and corrected MVCBCT+ of the CIRS thorax phantom. The cupping artefact is clearly visible in the uncorrected MVCBCT+. The ED in the corrected MVCBCT+ was accurately reconstructed in the phantom except in a small region at the lung to water interface. The error in mean ED was reduced from a maximum of $20-4 \%$ and the standard deviation (SD) of the ED in the water equivalent regions decreased from $13 \%$ to $3 \%$ (Table 1). After cupping and truncation artefact correction, errors in dose on the central leftright axis reduced from $6 \%$ (single beam) and $-2 \%$ (IMRT plan) to $0.5 \%$. The percentage of voxels with $\gamma<1$ increased from $74 \%$ to $99 \%$ (IMRT plan). Table 1 shows that the deviation in mean dose in the different volumes reduced from a maximum of 8$0 \%$ (IMRT plan).

Dose calculation on the uncorrected MVCBCT+ of the Rando thorax phantom led to an underestimation in dose in the center of the plan of $11 \%$ compared to $0 \%$ in the corrected MVCBCT+ 

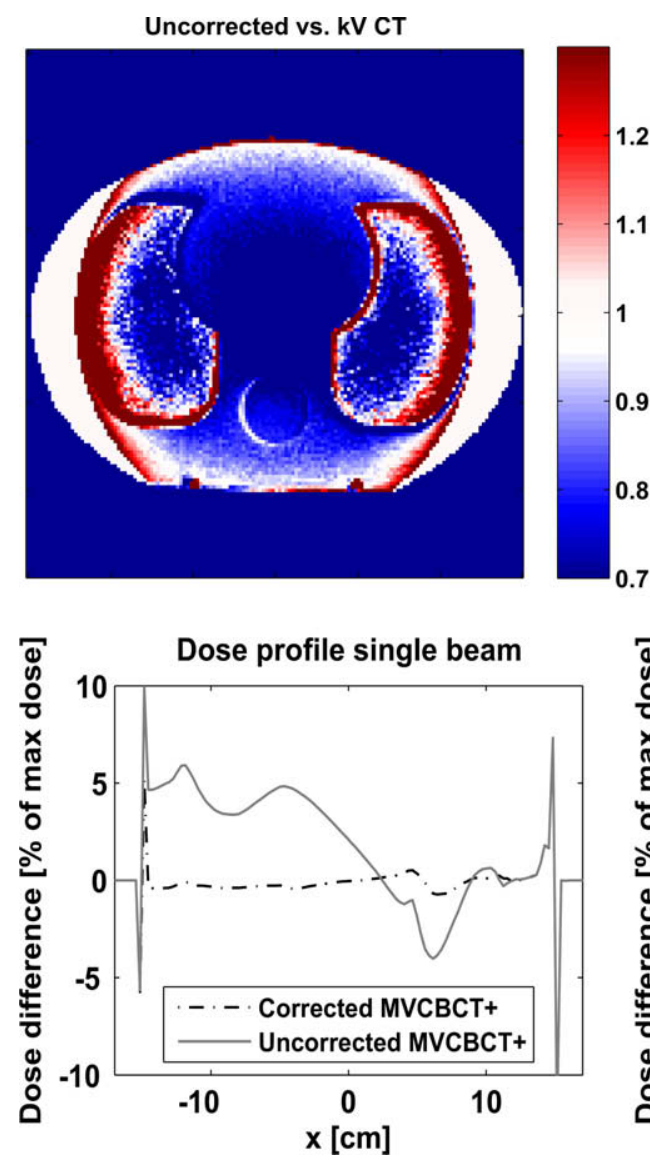
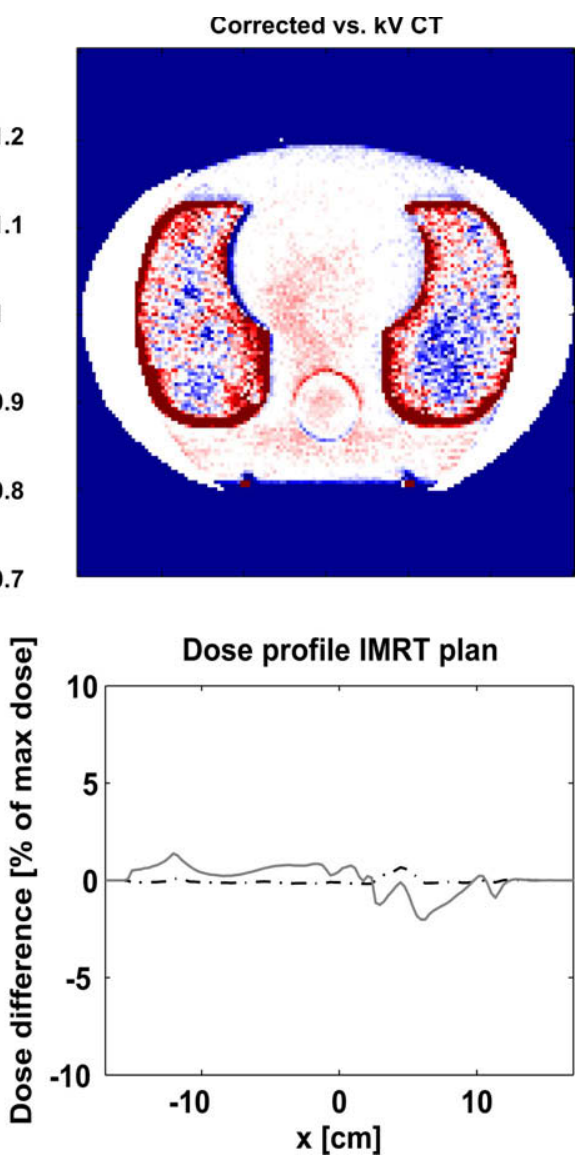

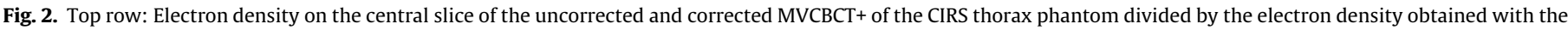

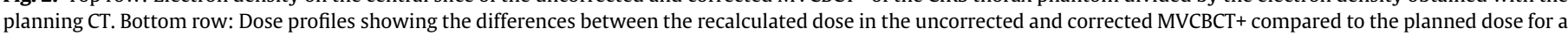
single beam and an IMRT plan.

Table 1

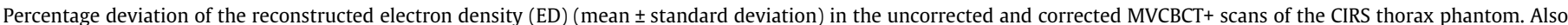
the deviation in mean dose for different volumes is indicated. The VOIs were defined in the water equivalent region.

\begin{tabular}{|c|c|c|c|c|c|c|c|c|}
\hline & Water & Left lung & Right lung & Bone & VOI $^{b}(4 \mathrm{~cm})$ & $\mathrm{VOI}^{\mathrm{b}}(5 \mathrm{~cm})$ & $\mathrm{VOI}^{\mathrm{b}}(6 \mathrm{~cm})$ & $\mathrm{VOI}^{\mathrm{b}}(7 \mathrm{~cm})$ \\
\hline \multicolumn{9}{|l|}{$E D^{a}$} \\
\hline Uncorrected & $-19 \pm 13 \%$ & $1 \pm 28 \%$ & $2 \pm 29 \%$ & $-20 \pm 7 \%$ & $-36 \pm 7 \%$ & $-36 \pm 7 \%$ & $-36 \pm 7 \%$ & $-36 \pm 7 \%$ \\
\hline Corrected & $1 \pm 4 \%$ & $-4 \pm 12 \%$ & $-1 \pm 12 \%$ & $4 \pm 2 \%$ & $4 \pm 3 \%$ & $3 \pm 4 \%$ & $2 \pm 5 \%$ & $2 \pm 5 \%$ \\
\hline \multicolumn{9}{|l|}{ Dose } \\
\hline Uncorrected & & $-1 \%$ & $8 \%$ & $3 \%$ & $4 \%$ & $3 \%$ & $2 \%$ & $2 \%$ \\
\hline Corrected & & $0 \%$ & $0 \%$ & $0 \%$ & $0 \%$ & $0 \%$ & $0 \%$ & $0 \%$ \\
\hline
\end{tabular}

a ED, electron density.

b VOI, volume of interest.

(Fig. 3, top row). The percentage of voxels with a $\gamma<1$ was $74 \%$ and $99 \%$, respectively.

The cupping artefact in the MVCBCT + of the homogeneous CIRS phantom caused an overestimation in dose which is clearly visible by the $105 \%$ contour (Fig. 3, bottom row). The iso-dose lines on the corrected MVCBCT+ overlapped perfectly with those of the planned dose distribution. Errors in dose in the VOIs reduced from $6 \%$ to $1 \%$ after correction and the percentage of voxels with $\gamma<1$ increased from $70 \%$ to $100 \%$.

Patients without changes in anatomy between the MVCBCT and the planning $C T$

No important anatomy changes were observed between the planning CT and the MVCBCT of lung patient 1 and an accurate registration was derived. In the uncorrected MVCBCT+ the ED is underestimated in the GTV and the surrounding lung, leading to an underestimation in mean dose of $10 \%$ and $11 \%$ in the GTV and planning target volume (PTV), respectively. After correction these mean doses were $1 \%$ and $3 \%$ larger than planned, respectively, and the percentage of voxels with $\gamma<1$ increased from $63 \%$ to $94 \%$. A good overlap with the planned iso-dose contours was observed (Fig. 4, left column). A dose profile in the right-left direction through the center of the GTV is shown. Similar results were obtained for lung cancer patient 2: after correction the mean GTV and PTV doses deviated only $1 \%$ from the planned doses.

No anatomical changes were observed between the MVCBCT of rectal cancer patient 1 and the planning $C T$ scan other than some small changes in the filling of the intestine. Dose calculations on the uncorrected MVCBCT+ lead to an overestimation of $4 \%$ and 
Uncorrected MVCBCT+

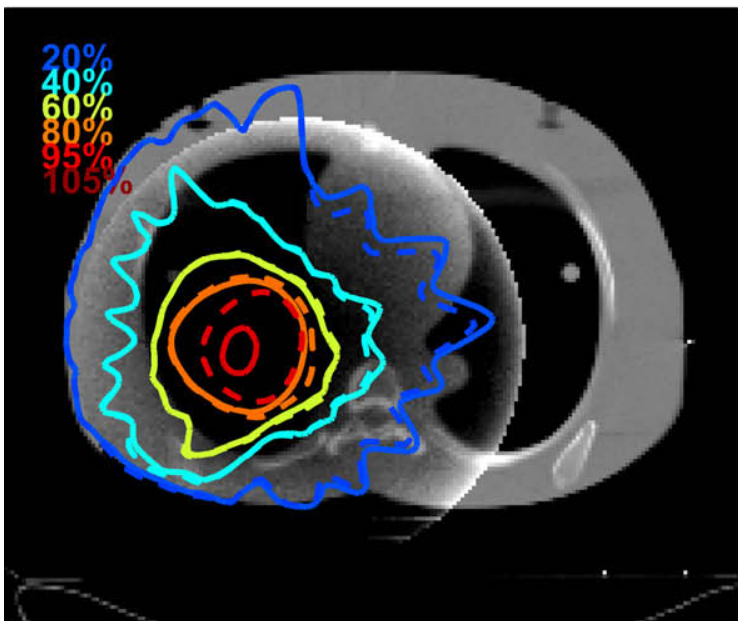

Uncorrected MVCBCT+

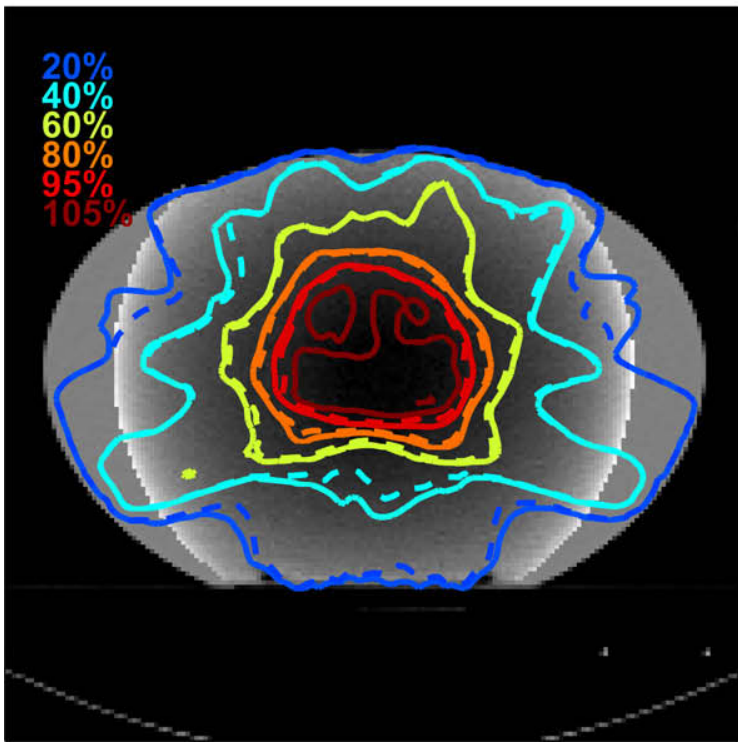

Corrected MVCBCT+

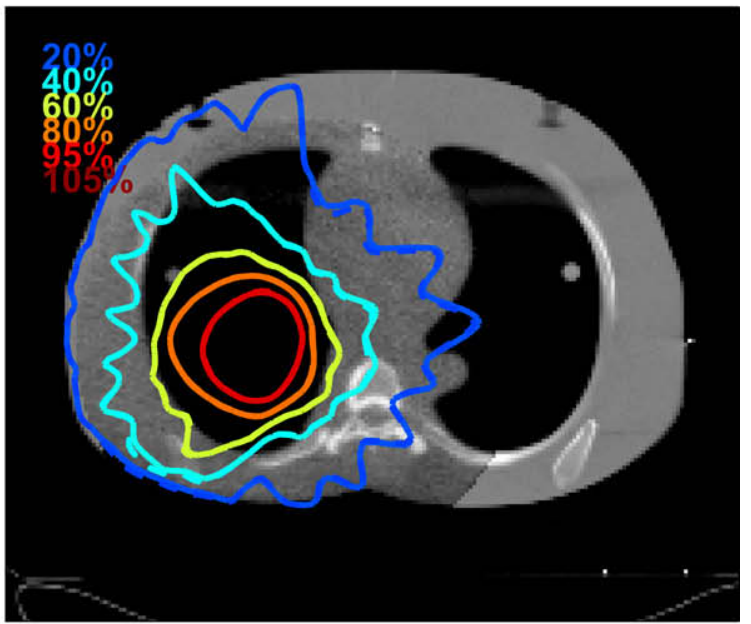

Corrected MVCBCT+

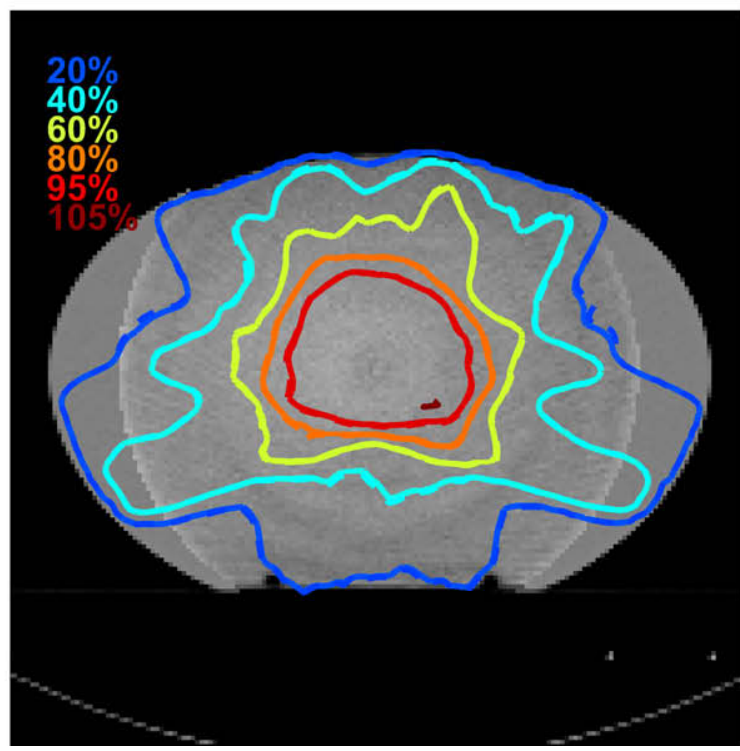

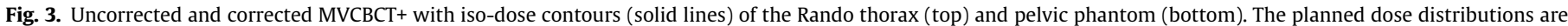
shown as dashed lines.

$3 \%$ of the mean GTV and PTV doses and to $81 \%$ of the voxels with $\gamma<1$. Dose calculation with the corrected MVCBCT + was accurate in $99 \%$ of the voxels $(\gamma<1)$ and the deviation in mean GTV and PTV doses was only $1 \%$. The dose profiles clearly show the overestimation in dose in the uncorrected MVCBCT+ (Fig. 4, right column).

Patients with changes in anatomy between the MVCBCT and the planning $C T$

Lung cancer patient 3 developed a vast amount of atelectasis in the left lung during treatment (Fig. 5, left column). Because the GTV was located ventrally the registration was focused on the ventral ribs and the mediastinum. A small misregistration near the dorsal part of the lung was accepted. The atelectasis caused an under dosage in the mediastinum and the 100\% iso-dose line reduced considerably leading to a $\gamma<1$ for only $75 \%$ of the voxels.

The second rectal cancer patient had a large air pocket in the rectum near the GTV at the time the planning CT scan was made. When the MVCBCT was acquired, the air was not present and dosimetric differences were expected (Fig. 5, right column). The $100 \%$ iso-dose lines calculated on the corrected MVCBCT+ shrunk considerably compared to the planned leading to a small under dosage to the GTV $(97 \%$ of voxels with $\gamma<1)$.

\section{Sensitivity analysis}

The effect of breathing motion was investigated by stitching the MVCBCT of lung patient 2 in the maximum inhale and exhale phases of the $4 \mathrm{D}$ CT scan. The position of the diaphragm varied $5 \mathrm{~mm}$ between both phases, but this had no effect on the dose calculations $(99.8 \%$ voxels with $\gamma<1)$. The weight loss simulations estimated that shrinkage of the patient diameter with $3.3 \mathrm{~cm}$ yields an underestimation in dose of $3-4 \%$ in the MVCBCT + . Artefacts were visible in the MVCBCT+.

\section{Discussion}

It was demonstrated that cupping and truncation artefacts in MVCBCT scans of the thorax and abdomen phantoms were adequately removed and that the corrected scans can be used for 

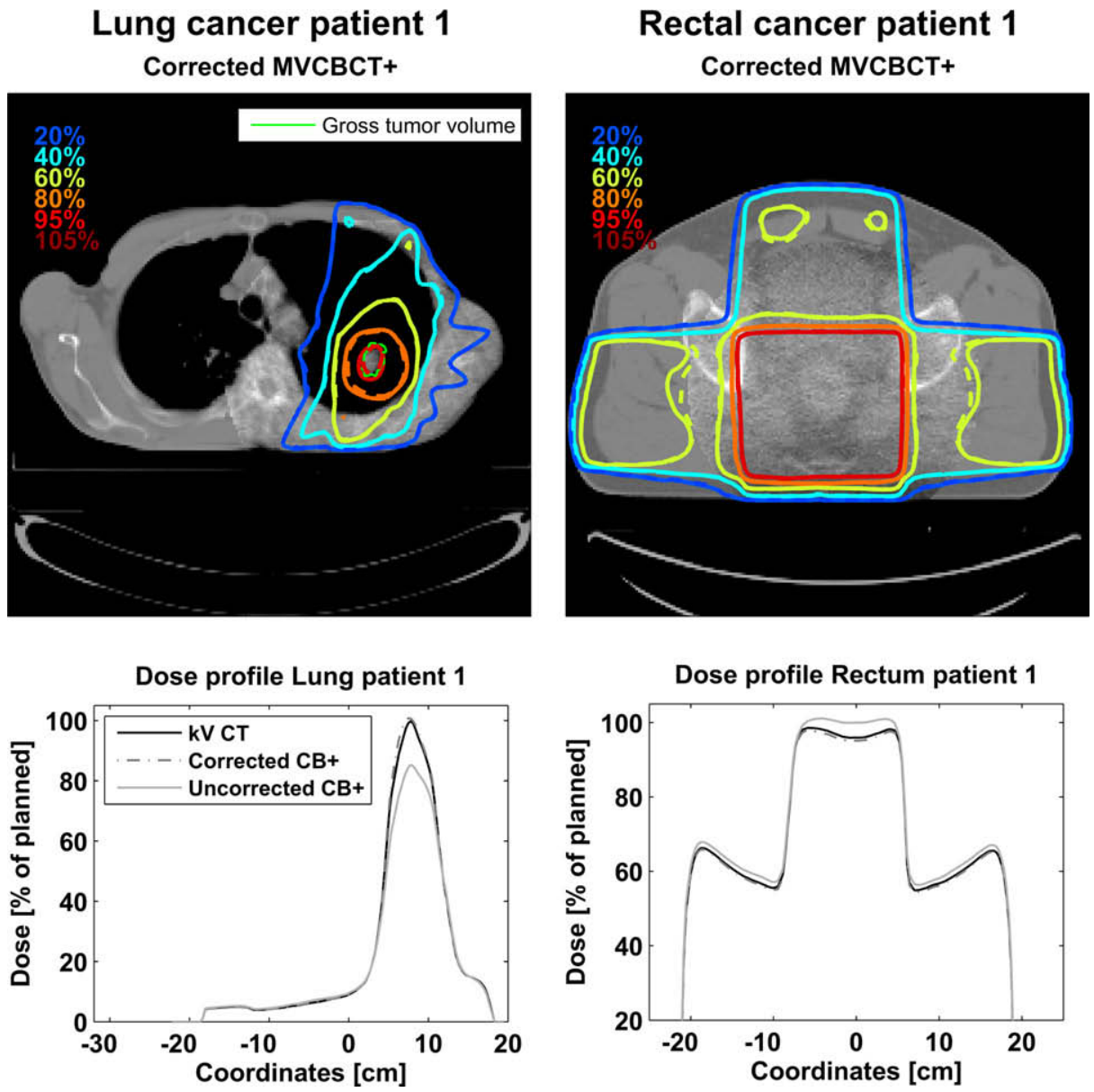

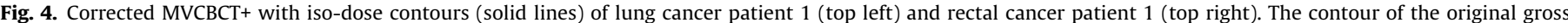

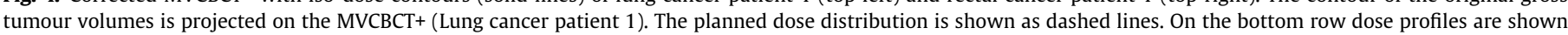
along the left-right direction through the center of the GTV.

accurate dose calculations of conformal, IMRT and SBRT treatment plans. The phantom study showed that dose calculations on uncorrected MVCBCT+ scans lead to large errors in dose of up to $11 \%$. With corrected MVCBCT+ scans deviations are obtained of only $1 \%$ in the mean VOI dose (99\% voxels with $\gamma<1$ ). The clinical feasibility of the method was demonstrated with MVCBCT scans of two lung and one rectal cancer patients. After cupping and truncation artefact correction the dose calculations were accurate (error in mean GTV dose $<1 \%$ ). This was expected because no important changes in anatomy were observed between the planning CT and MVCBCT scans. These six phantom and patient cases suggest that the proposed method is suitable to correct MVCBCT scans of the thorax and abdomen, that the corrected scans can be used for accurate dose calculations and therefore to study the 3D dose delivered during treatment. The feasibility of this method for head-and-neck patients was demonstrated previously [11]. The method can be fully automated and the current implementation takes approximately 30 min per MVCBCT+. Recent developments using graphical processing units (GPUs) could reduce the calculation time to less than 1 min [24].

Two additional cases were analyzed where the patient anatomy changed during therapy. It was evident from the images that the dose distributions would be affected. However, from inspection of the images alone it is difficult to estimate the magnitude of the dosimetric effect. Using our dose recalculation technique, we have shown that in both cases, the difference between the planned and delivered dose distribution was moderate. Both patients were treated with conformal techniques. It is expected that similar deviations will have a larger influence on SBRT or IMRT treatment plans. We demonstrated that dose recalculation using the MVCBCT+ is a valuable tool to quantify the influence of anatomical changes on the dose distribution.

The main advantages of dose recalculation with MVCBCT scans are that no additional scans need to be acquired if the MVCBCT has already been acquired for image guidance. The corrected MVCBCT+ is an accurate representation of the exact patient anatomy during the treatment and the effect of possible set-up deviations is already taken into account.

A sensitivity analysis was performed to investigate the effect of changes in anatomy that occur outside the FOV of the MVCBCT scanner. This analysis suggested that differences in lung volume and diaphragm position outside the FOV due to breathing are not of importance. This is because most relevant changes, e.g. diaphragm position near the GTV, occurred within the FOV of the cone-beam, if its isocenter coincides with that of the treatment beams. We demonstrated that unexpected weight loss does influence the accuracy of the dose calculations. The estimated effect is moderate though, considering that in our phantom simulations a decrease in patient diameter of $3.3 \mathrm{~cm}$ lead to an underestimation in dose of $4 \%$. This is not an important limitation of the method, because with such amount of weight loss, the real dose distribution will deviate so much from the planned dose distribution that a new planning CT must be acquired anyway to replan the treatment. To avoid the influence of anatomy changes outside the 


\section{Lung cancer patient 3}

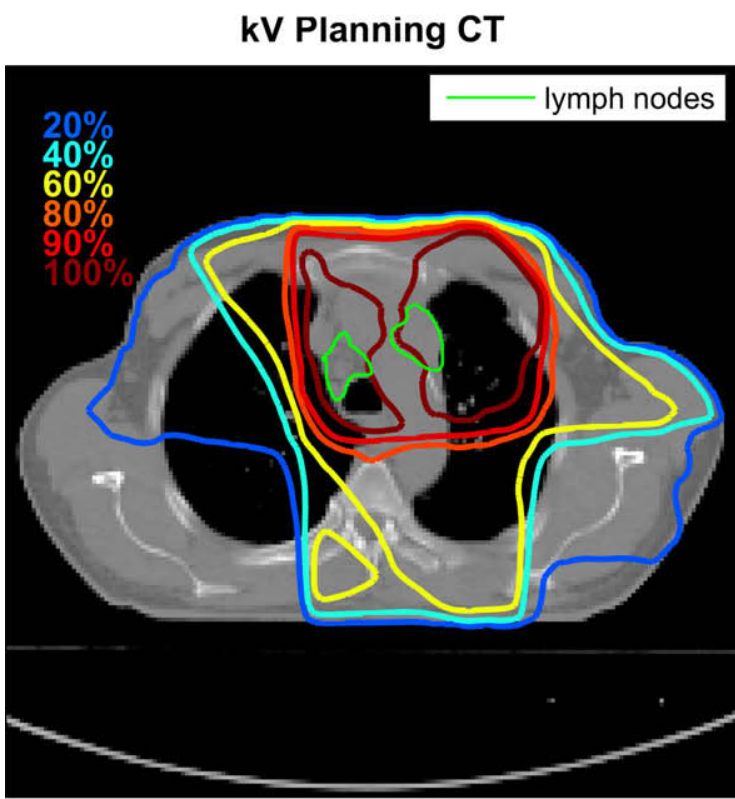

Corrected MVCBCT+

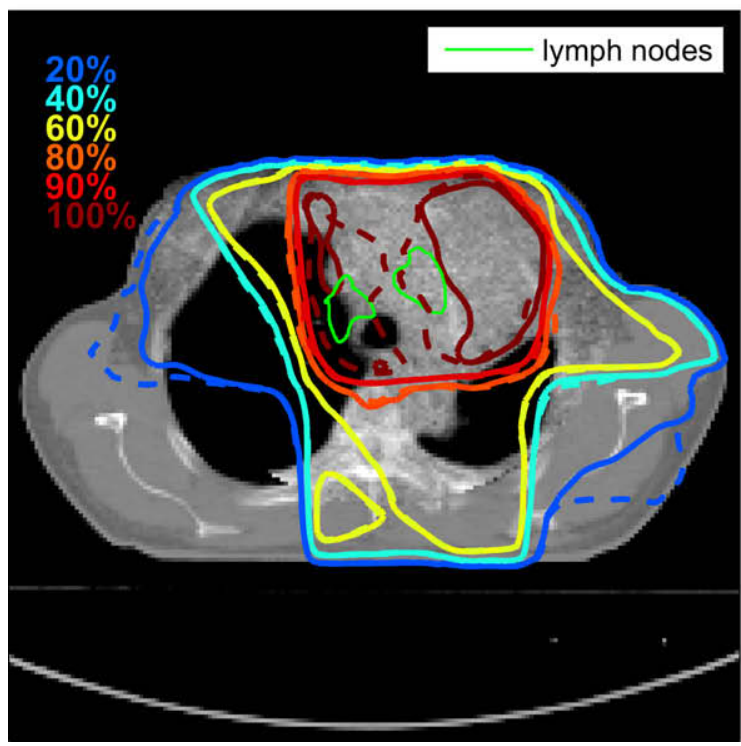

Rectal cancer patient 2

\section{kV Planning CT}

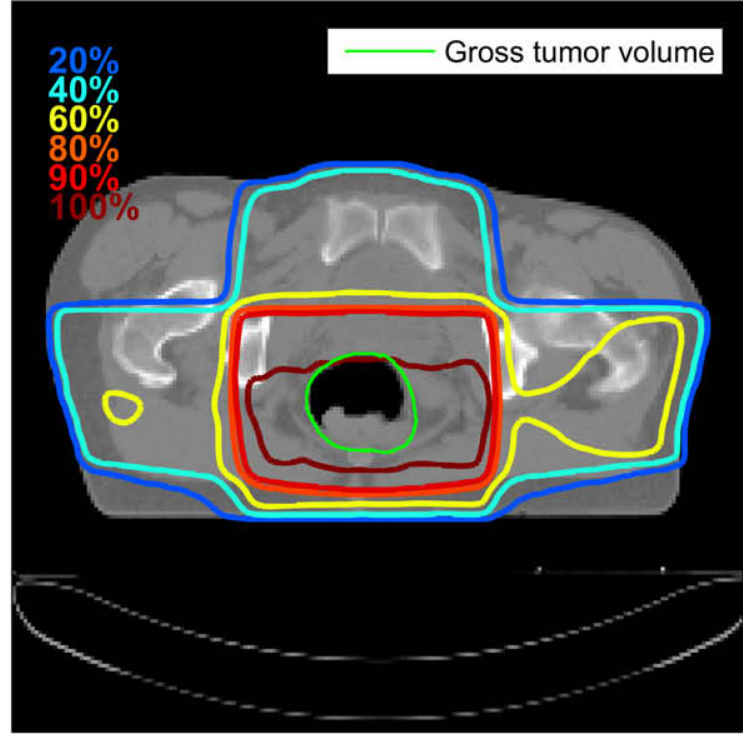

Corrected MVCBCT+

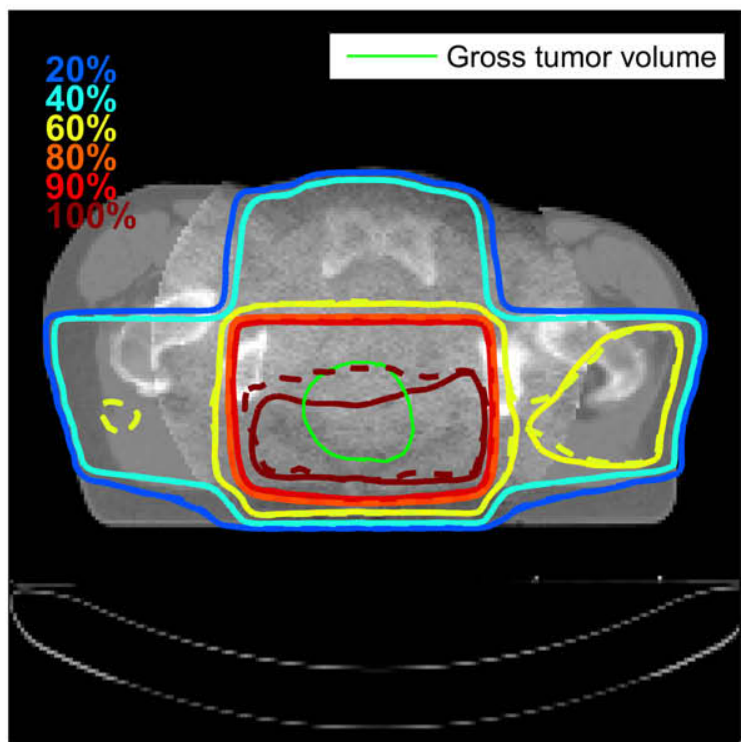

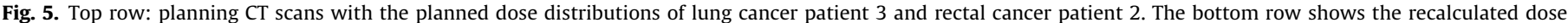
distributions on the corrected MVCBCT+. The planned dose distributions are shown as dashed lines.

FOV, the MVCBCT portal images could be acquired along an arc of $360^{\circ}$ with the imaging detector shifted laterally [17]. This was however not yet possible with the systems used in our clinic.

The MVCBCT scans of the patients were acquired with less dose than the phantoms. This could have affected the accuracy of ED reconstruction by approximately $1 \%[16]$ and therefore the effect on the dose calculations was negligible.

Aubry et al. proposed two separate methods to correct for cupping and truncation artefacts of low-dose MVCBCT scans of the head-and-neck and the abdomen and obtained an accuracy similar to the present method $[18,19]$. Maltz et al. applied a similar method as the current, but used scatter kernels derived with MC and obtained good results with MVCBCT scans of phantoms [17]. They also demonstrated the applicability of their method for kVCBCT. Also methods have been proposed to calibrate MV fan beam CT scans of the tomotherapy machine to ED. Because the scatter contribution is much smaller than for MVCBCT a simple CT number to ED calibration is sufficient for dose calculations [25]. To the best of our knowledge the present study is the first to demonstrate the feasibility of a single cupping and truncation artefact correction method for MVCBCT scans of both the thorax and the abdomen as an extension to head-and-neck, which was demonstrated previously [16].

\section{Conclusion}

A novel artefact correction method for MVCBCT scans of the thorax and abdomen was presented. The method extends a previous cupping artefact correction method, developed for 
head-and-neck, to remove truncation artefacts in cone-beam scans of patients that are larger than the FOV. The corrected cone-beam images of the three phantom and three patient cases were suitable for accurate dose calculations. The MVCBCT scans can be used to calculate the delivered patient dose distribution in 3D and to interpret the importance of anatomical changes observed with IGRT in terms of dose.

\section{Acknowledgements}

The authors thank Siemens Oncology Care Systems for technical and financial support. This study was performed within the framework of CTMM, the Center for Translational Molecular Medicine (www.ctmm.nl), project AIRFORCE number 030-103.

\section{References}

[1] Verellen D, Ridder MD, Linthout N, Tournel K, Soete G, Storme G. Innovations in image-guided radiotherapy. Nat Rev Cancer 2007;7:949-60.

[2] Gayou O, Miften M. Commissioning and clinical implementation of a megavoltage cone beam CT system for treatment localization. Med Phys 2007;34:3183-92.

[3] Mosleh-Shirazi MA, Evans PM, Swindell W, Webb S, Partridge M. A cone-beam megavoltage CT scanner for treatment verification in conformal radiotherapy. Radiother Oncol 1998;48:319-28.

[4] Yan D, Vicini F, Wong J, Martinez A. Adaptive radiation therapy. Phys Med Biol 1997;42:123-32.

[5] Dawson LA, Jaffray DA. Advances in image-guided radiation therapy. J Clin Oncol 2007;25:938-46.

[6] Chen J, Morin O, Aubin M, Bucci MK, Chuang CF, Pouliot J. Dose-guided radiation therapy with megavoltage cone-beam $\mathrm{CT} . \mathrm{Br} J$ Radiol 2006;79:S87-98.

[7] McDermott LN, Wendling M, Nijkamp J, et al. 3D in vivo dose verification of entire hypo-fractionated IMRT treatments using an EPID and cone-beam CT. Radiother Oncol 2008;86:35-42.

[8] Nijsten SM, van Elmpt WJ, Mijnheer BJ, et al. Prediction of DVH parameter changes due to setup errors for breast cancer treatment based on 2D portal dosimetry. Med Phys 2009;36:83-94.

[9] Hansen VN, Evans PM, Swindell W. The application of transit dosimetry to precision radiotherapy. Med Phys 1996;23:713-21.
[10] Cheung J, Aubry JF, Yom SS, Gottschalk AR, Celi JC, Pouliot J. Dose recalculation and the dose-guided radiation therapy (DGRT) process using megavoltage cone-beam CT. Int J Radiat Oncol Biol Phys 2009;74:583-92.

[11] van Elmpt W, Nijsten S, Petit S, Mijnheer B, Lambin P, Dekker A. 3D in vivo dosimetry using megavoltage cone-beam CT and EPID dosimetry. Int J Radiat Oncol Biol Phys 2009;73:1580-7.

[12] van Elmpt W, McDermott L, Nijsten S, Wendling M, Lambin P, Mijnheer B. A literature review of electronic portal imaging for radiotherapy dosimetry. Radiother Oncol 2008;88:289-309.

[13] Spies L, Ebert M, Groh BA, Hesse BM, Bortfeld T. Correction of scatter in megavoltage cone-beam CT. Phys Med Biol 2001;46:821-33.

[14] Partridge M, Ebert M, Hesse BM. IMRT verification by three-dimensional dose reconstruction from portal beam measurements. Med Phys 2002;29:1847-58.

[15] Morin O, Chen J, Aubin M, et al. Dose calculation using megavoltage conebeam CT. Int J Radiat Oncol Biol Phys 2007;67:1201-10.

[16] Petit SF, van Elmpt WJ, Nijsten SM, Lambin P, Dekker AL. Calibration of megavoltage cone-beam CT for radiotherapy dose calculations: correction of cupping artifacts and conversion of CT numbers to electron density. Med Phys 2008;35:849-65.

[17] Maltz JS, Gangadharan B, Bose S, et al. Algorithm for X-ray scatter, beamhardening, and beam profile correction in diagnostic (kilovoltage) and treatment (megavoltage) cone beam CT. IEEE Trans Med Imaging 2008;27:1791-810.

[18] Aubry JF, Cheung J, Morin O, Gottschalk A, Beaulieu L, Pouliot J. Correction of megavoltage cone-beam CT images of the pelvic region based on phantom measurements for dose calculation purposes. J Appl Clin Med Phys 2009; $10: 2852$

[19] Aubry JF, Pouliot J, Beaulieu L. Correction of megavoltage cone-beam CT images for dose calculation in the head and neck region. Med Phys 2008;35:900-7.

[20] van Zijtveld M, Dirkx M, Heijmen B. Correction of conebeam CT values using a planning CT for derivation of the "dose of the day". Radiother Oncol 2007:85:195-200.

[21] Ford EC, Chang J, Mueller K, et al. Cone-beam CT with megavoltage beams and an amorphous silicon electronic portal imaging device: potential for verification of radiotherapy of lung cancer. Med Phys 2002;29:2913-24.

[22] Sillanpaa J, Chang J, Mageras G, et al. Low-dose megavoltage cone-beam computed tomography for lung tumors using a high-efficiency image receptor. Med Phys 2006;33:3489-97.

[23] Pouliot J, Bani-Hashemi A, Chen J, et al. Low-dose megavoltage cone-beam CT for radiation therapy. Int J Radiat Oncol Biol Phys 2005;61:552-60.

[24] Noel PB, Walczak AM, Xu J, Corso JJ, Hoffmann KR, Schafer S. GPU-based cone beam computed tomography. Comput Methods Programs Biomed 2009.

[25] Langen KM, Meeks SL, Poole DO, et al. The use of megavoltage CT (MVCT) images for dose recomputations. Phys Med Biol 2005;50:4259-76. 